\title{
The Impact of a Concussion Clinic on Family Medicine Resident Education
}

\author{
Theodore A. Ogren, MD | Alexander C. Knobloch, MD, CAQSM
}

PRIMER. 2019;3:12.

Published: 4/12/2019 | DOI: 10.22454/PRiMER.2019.465841

\section{Abstract}

Introduction: Given that primary care has been found to be the most likely point of entry into the health care system for concussion patients, diagnosis and management of concussions are vital to the education of family medicine physicians. Studies of primary care residencies reveal a self-perceived deficiency in sports medicine education. This project was designed to determine the effect of a concussion management clinic on family medicine residents' confidence in and personal value placed on diagnosing and managing concussions.

Methods: This project was completed in a family medicine residency as a retrospective evaluation of an educational improvement project during the 2016-2017 academic year. The intervention involved implementation of a structured clinical experience focused on management of concussions. Residents were surveyed preintervention, as well as 5 and 11 months postintervention, measuring resident exposure to and confidence in diagnosing and managing concussions.

Results: Residents demonstrated significantly increased confidence in diagnosing concussions and managing complicated concussions following the intervention (both $F[2,84]=3.56, P=0.03$ ). Post hoc analysis indicated the statistical difference was found between preintervention measures and 1 academic year later. The number of concussions seen positively correlated with resident confidence in both diagnosing and managing concussions while personal value remained high.

Conclusions: This project is the first to evaluate the impact of a concussion clinic on resident education, demonstrating significant improvement in multiple areas of concussion education. Replicating the project in other family medicine residencies could be beneficial in determining if this clinic education model improves resident outcomes in other residencies.

\section{Introduction}

Concussion management has proven to be an important topic in family medicine education as concussions, or mild traumatic brain injuries, occur in a wide variety of sports and occupations, ${ }^{1,2}$ with many states requiring medical professional clearance to return to play in high school sports. ${ }^{3,4}$ Additionally, primary care is the most likely point of entry into the health care system for concussion patients, ${ }^{5}$ while studies on primary care residents have revealed a deficiency in concussion management and diagnostic knowledge ${ }^{6}$ as well as overall sports medicine education. ${ }^{7-10}$

A concussion referral clinic within a family medicine residency would be ideally suited to centralize patients into a unique educational experience by increasing concussion patient numbers and providing a structured setting for faculty to evaluate residents. While this type of resident educational experience has been seen in similar referralbased residency procedure clinics, ${ }^{11}$ literature is sparse in studies evaluating the educational outcomes of such experiences. 
In summer 2016, a concussion referral clinic was established within a family medicine residency, comprised of 12 residents per postgraduate year, embedded within a community-based hospital in the Southeastern United States. The concussion clinic took place within a sports medicine specialty clinic that was physically located within the family medicine residency. Concussion patients were previously managed within their individual primary care clinics. The concussion clinic consolidated continuity of care for virtually all new concussion patients at the hospital, as the emergency department and each primary care office were provided a pathway to refer patients with a goal of each patient being seen in the referral clinic within 72 hours of initial presentation. All referral sources were provided with a handout to give to patients contact information for the concussion clinic, as well as initial management recommendations for concussion patients. Patients were excluded from enrollment and sent for specialty evaluation in cases of open head trauma, radiologic abnormalities on imaging indicating trauma, or if the concussion had occurred more than 2 weeks prior to presentation.

Over the course of the first academic year during which the concussion clinic was implemented, residents cared for 57 concussion patients. To provide exposure to a variety of concussion evaluation tools and in the patient settings they might be most helpful, residents used the Sport Concussion Office Assessment Tool (SCOAT), ${ }^{12}$ Child-SCAT3, ${ }^{13}$ and the Military Acute Concussion Evaluation (MACE). ${ }^{14}$ Patients were scheduled for follow up within the clinic until completion of a return-to-play protocol or until they had demonstrated prolonged or atypical symptoms requiring specialty referral. We hypothesized that implementation of this concussion clinic would increase residents' confidence in diagnosing and managing concussions. Additionally, we hypothesized that residents place a high personal value on these clinical skills, and that this high value would remain unchanged or further increase with their participation in the concussion clinic.

\section{Methods}

During the 2016-2017 academic year, an anonymous online survey was sent to family medicine residents at a community-based hospital. The survey was sent before implementation of the clinic, using a Likert-type scale to establish a baseline of resident confidence in diagnosing and managing concussions, their perceived value of these clinical skills, and an estimate of the number of concussion cases they have treated throughout their medical careers. This survey was resent to residents 5 and 11 months after implementation of the clinic, the latter coinciding with completion of the academic year.

The differences between survey results were compared using analysis of variance (ANOVA). Additionally, the number of concussions seen was tested for association with resident confidence and value using a Pearson correlation. Data sets were analyzed using SPSS 24.0 (IBM, Armonk, NY). The Air Force Office of Research Oversight and Compliance granted this study an institutional review board exemption.

\section{Results}

Residents demonstrated a statistically significant increase in confidence in diagnosing concussions postintervention $(F[2,84]=3.56, P=0.03$; Table 1$)$. Additionally, residents demonstrated a statistically significant increase in confidence in managing complicated concussions $(F[2,84]=3.56, P=0.03)$. Post hoc analysis indicated the statistical difference existed between the mean responses preintervention and at 11 months, but not between preintervention and 5 months. No statistical difference was demonstrated postintervention in the high value residents placed on concussion management topics (Table 1).

Further analysis of the data showed the number of concussions seen (Table 2) positively correlated with both resident confidence and value placed on diagnosing concussion, managing both uncomplicated and complicated concussions, and advising athletes on return to play (Table 3).

\section{Discussion}

Implementing a concussion referral clinic within a family medicine residency's sports medicine clinic was 
successful in increasing resident confidence in diagnosing and managing concussions. The statistical difference in confidence was between preintervention and 11 months, and not 5 months. This trend may suggest that increased exposure over time is essential to increasing confidence; however, given our analysis of the data using mean survey responses and resident exposure to the concussion clinic occurring only during a specific clinical rotation during the academic year, this trend may more likely be due to increasing participation of the residents in the clinic as the year progressed.

As hypothesized, residents placed high value on these clinical skills, and this value was maintained postintervention. Thus, in light of these valued skills, increased confidence in concussion diagnosis and management is a first step to increasing willingness of residents to provide optimal concussion care beyond residency.

Although this project was not designed to directly assess patient outcomes, we hypothesize that increased confidence and increased exposure to concussions could lead to better, more standardized treatment. By establishing a central location for concussion management, future studies on patient outcomes would be easier to perform, allowing further opportunities to monitor outcomes. New studies could be conducted to gauge how effective residents were in managing concussions before and after the clinic's implementation by studying various patient-oriented outcomes or provider standard-of-care reviews, as it is difficult to conclude whether the statistically significant increases in our mean survey responses were clinically or educationally meaningful without such outcome-based measures.

Although results showed a statistically significant increase in resident confidence, the study lacked a control that would isolate the concussion clinic and not routine residency curriculum as the reason for this increase in confidence. The positive correlation between number of concussions seen and our measured variables would suggest this increase was impacted by a clinic experience designed to increase concussion patient exposure. As surveys were anonymous, participants' responses were not linked across time points, thus this study was limited to using a one-way ANOVA to best analyze results, despite having to violate the assumption of independence between samples. However, as the analysis utilized the same small cohort of residents, findings were more likely to represent improvements in confidence rather than demographic shifts. As with all self-reported surveys, bias is possible and a social desirability bias could have inclined residents to respond favorably.

\section{Conclusion}

This project was the first to evaluate the impact of a concussion clinic on resident education and is a promising step toward improving concussion education in family medicine and other primary care residencies. Additionally, this project further validates the use of specialty clinics within family medicine residencies as an educational tool. Future studies could be directed toward adopting a similar clinic at other primary care residencies, with efforts made to either include a full academic year of preintervention monitoring or a separate control residency program if multiple sites could be studied.

\section{Tables and Figures}


Table 1: Effects of a Concussion Referral Clinic on Family Medicine Resident Concussion Education

\begin{tabular}{|c|c|c|c|c|c|c|}
\hline & \multicolumn{3}{|c|}{$\begin{array}{l}\text { Mean Survey Responses } \\
\text { (SD) }\end{array}$} & \multirow[b]{2}{*}{$F(P)$} & \multirow{2}{*}{$\begin{array}{l}\text { Mean Difference } \\
\text { Pre- and } \\
\text { 5-Months } \\
\text { Postintervention } \\
(P)\end{array}$} & \multirow{2}{*}{$\begin{array}{l}\text { Mean Difference } \\
\text { Pre- and } \\
11 \text { Months } \\
\text { Postintervention } \\
(P)\end{array}$} \\
\hline & $\begin{array}{l}\text { Preintervention } \\
\qquad(\mathrm{n}=\mathbf{3 4})\end{array}$ & $\begin{array}{c}5 \text { Months } \\
\text { Postintervention } \\
(n=29)\end{array}$ & $\begin{array}{c}11 \text { Months } \\
\text { Postintervention } \\
(n=24)\end{array}$ & & & \\
\hline \multicolumn{7}{|c|}{ Confidence (Measure of Self-Efficacy) } \\
\hline 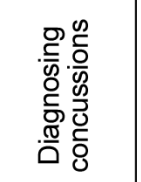 & $3.94(0.81)$ & $4.17(0.49)$ & $4.42(0.65)$ & $\begin{array}{l}3.56 \\
(0.03) \\
b\end{array}$ & $0.23(0.37)$ & $0.48(0.025)$ \\
\hline 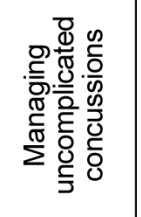 & $4.09(0.71)$ & $4.21(0.56)$ & $4.38(0.58)$ & $\begin{array}{c}1.47 \\
(0.24)\end{array}$ & ns & ns \\
\hline 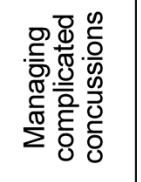 & $2.76(0.99)$ & $3.07(1.03)$ & $3.46(0.888)$ & $\begin{array}{c}3.56 \\
(0.03) \\
b\end{array}$ & $0.3(0.44)$ & $0.69(0.025)$ \\
\hline 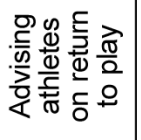 & $3.53(0.96)$ & $3.79(0.86)$ & $4.04(0.81)$ & $\begin{array}{l}2.38 \\
(0.1)\end{array}$ & ns & ns \\
\hline \multicolumn{7}{|c|}{ Value (Measure of Response Efficacy) } \\
\hline 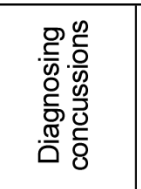 & $4.68(0.48)$ & $4.76(0.44)$ & $4.75(0.44)$ & $\begin{array}{c}0.31 \\
(0.73)\end{array}$ & ns & ns \\
\hline 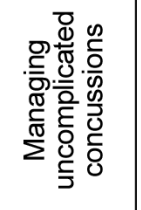 & $4.68(0.48)$ & $4.76(0.44)$ & $4.75(0.44)$ & $\begin{array}{c}0.31 \\
(0.73)\end{array}$ & ns & ns \\
\hline 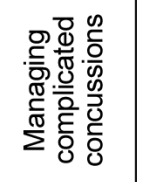 & $4.06(0.65)$ & $4.18(0.61)$ & $4.08(0.72)$ & $\begin{array}{c}0.27 \\
(0.76)\end{array}$ & ns & ns \\
\hline 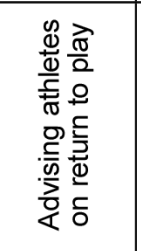 & $4.65(0.49)$ & $4.72(0.46)$ & $4.71(0.47)$ & $\begin{array}{c}0.24 \\
(0.79)\end{array}$ & ns & ns \\
\hline
\end{tabular}

aEach item measured on 1 (negative) to 5 (positive) scale.

bTukey post hoc analysis revealed that the statistical difference existed between the mean responses pre-intervention and at 11 months. Abbreviation: ns, not significant. 
Table 2: Resident Estimates of Number of Concussion Patients Seen During Course of Medical Career

\begin{tabular}{|c|c|}
\hline & $\begin{array}{c}\text { Number of Concussion Patients Seen } \\
\text { Mean } \pm \text { SD (Min-Max) }\end{array}$ \\
\hline Preintervention $(n=34)$ & $11.2 \pm 15.0(1-75)$ \\
\hline 5 months postintervention $(n=29)$ & $10.9 \pm 11.0(1-45)$ \\
\hline 11 months postintervention $(n=24)$ & $14.4 \pm 11.8(3-55)$ \\
\hline
\end{tabular}

Table 3: Correlations Between the Number of Concussions Seen and Family Medicine Resident Concussion Education

\begin{tabular}{|c|c|c|c|}
\hline & $\mathbf{n}$ & $\begin{array}{c}\text { Pearson } \\
\text { Correlation } \\
\text { Coefficient (r) }\end{array}$ & Sig (2-tailed) \\
\hline \multicolumn{4}{|c|}{ Confidence (Measure of Self-Efficacy) } \\
\hline Diagnosing concussions & 87 & 0.356 & 0.001 \\
\hline Managing uncomplicated concussions & 87 & 0.314 & 0.003 \\
\hline Managing complicated concussions & 87 & 0.414 & $<0.001$ \\
\hline Advising athletes on return to play & 87 & 0.366 & $<0.001$ \\
\hline \multicolumn{4}{|c|}{ Value (Measure of Response Efficacy) } \\
\hline Diagnosing concussions & 86 & 0.234 & 0.029 \\
\hline Managing uncomplicated concussions & 87 & 0.242 & 0.024 \\
\hline Managing complicated concussions & 87 & 0.224 & 0.038 \\
\hline Advising athletes on return to play & 87 & 0.242 & 0.024 \\
\hline
\end{tabular}

\section{Acknowledgments}

The authors thank Dr Christy Ledford and the Military Primary Care Research Network for help with statistical design, analysis, and dissemination of this project; Dr Erin Reddis for help implementing this project; and Drs Christopher Ledford and Sabrina Silver for their support and guidance.

Presentations: This project was presented at the 2017 Uniformed Services Academy of Famiy Physicians Annual Meeting, Seattle, WA, as a study in progress poster and then as a completed project at the 2018 Uniformed Services Academy of Family Physicians Annual Meeting, Ponte Vedra Beach, FL as a podium presentation.

Disclosures: The opinions and assertions contained herein are the private views of the authors and should be construed neither as official nor as reflecting the views of the the US Air Force, US Department of Defense, or the Uniformed Services University.

\section{Corresponding Author}

Theodore A. Ogren, MD

Langley Family Health Clinic, 633rd Medical Group, 77 Nealey Ave, Building 225, Langley AFB, VA, 23665.

703-625-6951. Fax: 757-225-7630.

theodore.a.ogren.mil@mail.mil

\section{Author Affiliations}

Theodore A. Ogren, MD - Langley Family Health Clinic, Joint Base Langley-Eustis, VA

Alexander C. Knobloch, MD, CAQSM - David Grant Medical Center Family Medicine Residency, Travis AFB, CA 


\section{References}

1. Marar M, Mcllvain NM, Fields SK, Comstock RD. Epidemiology of concussions among United States high school athletes in 20 sports. Am J Sports Med. 2012;40(4):747-755. https://doi.org/10.1177 /0363546511435626

2. Laker SR. Epidemiology of concussion and mild traumatic brain injury. PM R. 2011;3(10)(suppl 2):S354-S358. https://doi.org/10.1016/j.pmrj.2011.07.017

3. Albano AW Jr, Senter C, Adler RH, Herring SA, Asif IM. The legal landscape of concussion: implications for sports medicine providers. Sports Health. 2016;8(5):465-468. https://doi.org/10.1177/1941738116662025

4. Tomei KL, Doe C, Prestigiacomo CJ, Gandhi CD. Comparative analysis of state-level concussion legislation and review of current practices in concussion. Neurosurg Focus. 2012;33(6):E11: 11-19. https://doi.org /10.3171/2012.9.FOCUS12280

5. Arbogast KB, Curry AE, Pfeiffer MR, et al. Point of health care entry for youth with concussion within a large pediatric care network. JAMA Pediatr. 2016;170(7):e160294. https://doi.org/10.1001 /jamapediatrics.2016.0294

6. Mann A, Tator $\mathrm{CH}$, Carson JD. Concussion diagnosis and management: knowledge and attitudes of family medicine residents. Can Fam Physician. 2017;63(6):460-466.

7. Amoako AO, Amoako AB, Pujalte GG. Family medicine residents' perceived level of comfort in treating common sports injuries across residency programs in the United States. Open Access J Sports Med. 2015;6:81-86. https://doi.org/10.2147/OAJSM.S71457

8. Lebrun CM, Mrazik M, Prasad AS, et al. Sport concussion knowledge base, clinical practises and needs for continuing medical education: a survey of family physicians and cross-border comparison. Br J Sports Med. 2013;47(1):54-59. https://doi.org/10.1136/bjsports-2012-091480

9. Zonfrillo MR, Master CL, Grady MF, Winston FK, Callahan JM, Arbogast KB. Pediatric providers' self-reported knowledge, practices, and attitudes about concussion. Pediatrics. 2012;130(6):1120-1125. https://doi.org /10.1542/peds.2012-1431

10. Demorest RA, Bernhardt DT, Best TM, Landry GL. Pediatric residency education: is sports medicine getting its fair share? Pediatrics. 2005;115(1):28-33. https://doi.org/10.1542/peds.2004-0266

11. Dor K. Utilization of a referral-based procedure clinic to improve procedure training and assessment in a family medicine residency. J Grad Med Educ. 2014;6(2):358-359. https://doi.org/10.4300/JGME-D-14-00063.1

12. Patricios J, Collins R, Branfield A, Roberts $C$, Kohler R. The sports concussion note: should SCAT become SCOAT? Br J Sports Med. 2012;46(3):198-201. https://doi.org/10.1136/bjsports-2011-090386

13. Child SCAT3: Sport Concussion Assessment Tool. Br J Sports Med. 2013;47(5):263.

14. McCrea M, Guskiewicz K, Doncevic S, et al. Day of injury cognitive performance on the Military Acute Concussion Evaluation (MACE) by US military service members in OEF/OIF. Mil Med. 2014;179(9):990-997. https://doi.org/10.7205/MILMED-D-13-00349

Copyright $@ 2019$ by the Society of Teachers of Family Medicine 